\title{
The Time Course of Changes in Retinal Vessel Diameter in Response to Differing Durations of Flicker Light Provocation
}

\author{
Rebekka Heitmar and Robert J. Summers \\ School of Life and Health Sciences, Aston University, Birmingham, United Kingdom
}

Correspondence: Rebekka Heitmar, Aston University, Aston Triangle, Birmingham, B4 7ET, UK;

R.Heitmar1@aston.ac.uk.

Submitted: April 13, 2015

Accepted: September 30, 2015

Citation: Heitmar R, Summers RJ. The time course of changes in retinal vessel diameter in response to differing durations of flicker light provocation. Invest Ophthalmol Vis Sci. 2015;56:7581-7588. DOI:10.1167/ iovs.15-17089
Purpose. To determine the response of retinal vessels to differing durations of flicker light (FL) stimulation.

Methods. We recorded retinal arterial and venous vessel dilation to $12.5 \mathrm{~Hz}$ FL provocation of varying duration $(5,7,10$, and 20 seconds) in 12 healthy young individuals (age range, 26-45 years). All participants underwent a full ocular examination including IOP and blood pressure measurements.

Results. Maximum dilation (MD) did not show a significant dependence on flicker duration in arteries, whereas maximum constriction (MC) did. In veins, however, MD significantly increased with flicker duration. Approximately $80 \%$ to $90 \%$ of MD in arteries is reached within 10 seconds of FL stimulation.

Conclusions. The vast majority of arterial dilatory capacity is reached within 10 seconds of FL stimulation even though venous dilation continues strongly. Since MC of arteries shows a significant dependence on flicker duration, measurements at two different durations can provide more information about the retinal vascular system than at a single flicker duration alone.

Keywords: retinal vessel dilation, flicker light stimulation, flicker duration
$F^{1 / \alpha e n}$ licker light (FL) provocation is now a widely-established noninvasive means of assessing the autoregulation, endothelial function, and reactivity of retinal vessels by continuous measurement of retinal vessel diameters. Numerous groups have examined its potential for diagnostic applications in diabetes,${ }^{1-5}$ cardiovascular disease, ${ }^{6,7}$ and glaucoma ${ }^{8-10}$ (see Heitmar and Summers ${ }^{11}$ for a review). Over time a standard protocol has emerged comprising 50 seconds baseline measurement (continuous illumination) followed by three cycles of 20 seconds, $12.5 \mathrm{~Hz} \mathrm{FL}$, and 80 seconds recovery (total measurement time $=350$ seconds) ${ }^{12}$ Prior to the introduction of this protocol, researchers have explored different FL frequencies in order to identify the flicker frequencies that lead to maximum vessel dilation ${ }^{13}$ and found flicker frequencies in the range 4 to $16 \mathrm{~Hz}$ provoked strong dilatory responses in both arteries and veins.

In order to obtain a good measurement, clear media, good fixation, and maximum pupil dilation are mandatory. Where most of these conditions can be achieved, the total length of the measurement demands good compliance, which is especially difficult during FL provocation. Since the introduction of the "standard protocol" outlined above there has been no systematic effort to investigate the effects of varying duration of FL provocation. While some groups ${ }^{1,14}$ use a 3-flicker-cycle protocol where each flicker cycle has a different duration (16, 32 , and 64 seconds) the flicker frequency $(8 \mathrm{~Hz})$ and method of flicker provocation (flicker is spectrally distinct from measurement illumination) make the results difficult to compare with those obtained with the standard protocol where the flicker is obtained by completely interrupting the illumination at $12.5 \mathrm{~Hz}$ and a single duration is measured in one setting. An early study ${ }^{15}$ compared flicker durations of 10 and 30 seconds but the protocol for each flicker duration was different; for FL durations of 10 seconds five cycles of flicker were separated by 80 seconds of baseline measurements, whereas for durations of 30 seconds only three cycles were measured and each cycle was separated by 120 seconds. Analysis was carried out on the vessel diameters averaged over the flicker cycles (three or five). Although there was a significant association between flicker duration and vessel dilation on average the maximum dilation of arteries increased by only $20 \%$ (2.2\% above baseline diameter for 10 seconds to $2.6 \%$ above baseline diameter for 30 seconds), whereas the maximum dilation for veins increased by $75 \%$ $(2.35 \%-4.15 \%)$. From visual inspection of the flicker profile it is apparent that during the FL provocation the retinal arterioles in most healthy subjects exhibit a 2-stage reaction. The first stage is an acute phase (5-10 seconds duration) where the vessel rapidly dilates, followed by a plateau phase (maintenance phase) where dilation barely increases at all (e.g., see dotted line in top panel of Fig. 2). Upon flicker cessation the arteries rapidly constrict (to below the level of baseline) before more gently returning to the baseline level. In comparison to arteries, veins behave quite differently (see dotted line in bottom panel of Fig. 2); they continue to dilate after flicker cessation and return much less rapidly to their baseline diameter without constricting below it. What remains unknown is the diameter profile after flicker cessation at much shorter flicker durations (e.g., 5 seconds). It follows that a shorter FL duration would suffice to evaluate the acute phase of retinal arterial dilation but would also allow evaluation of the recovery phase or return-to- 

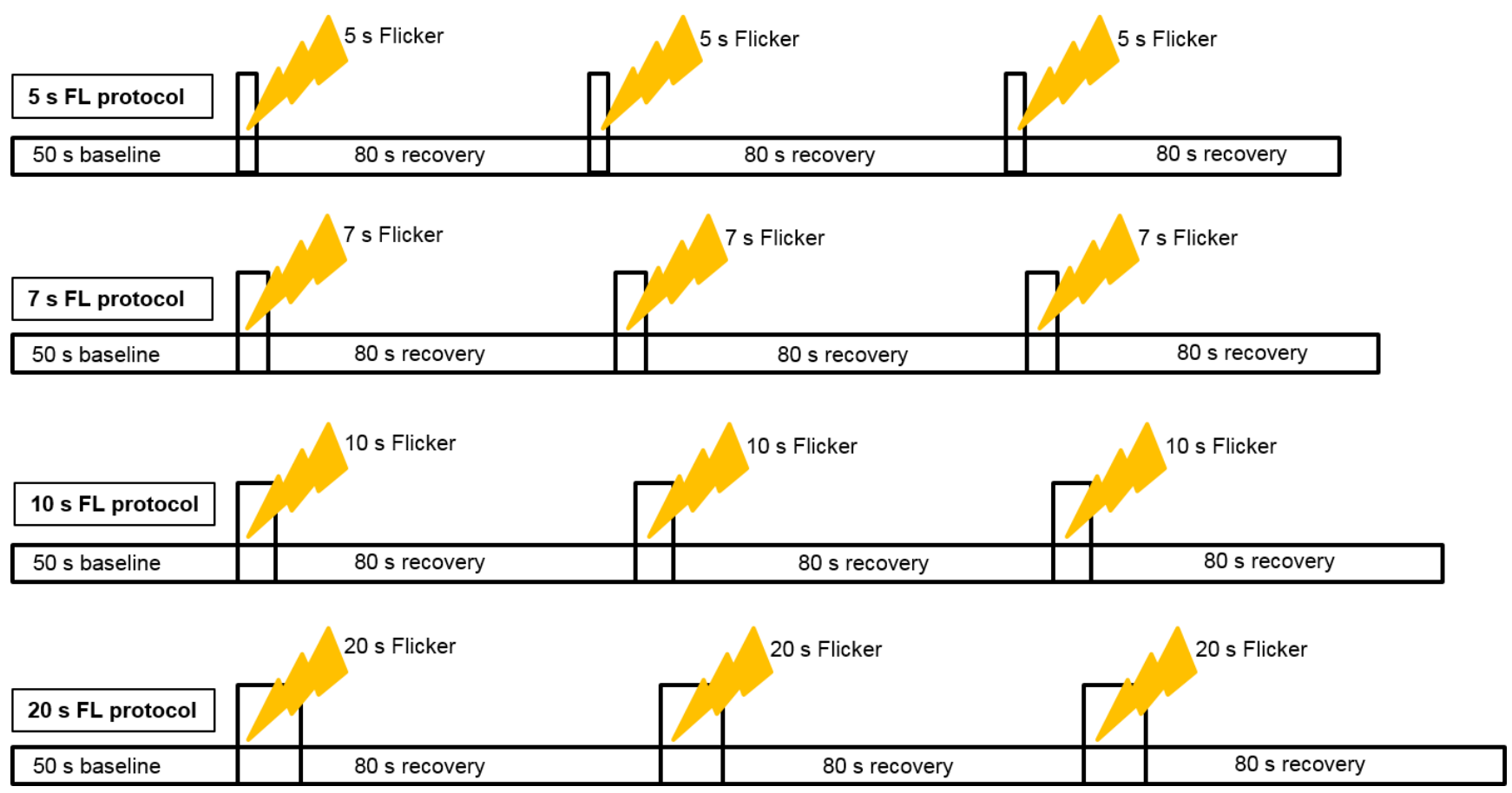

Figure 1. Schematic diagram of the different flicker light protocols used. From top to bottom: 5-, 7-, 10-, and 20-seconds FL durations.

normal, which may behave differently at shorter durations (e.g., arteries may not constrict below baseline diameter, which would likely be coupled with a lack of continued dilation in veins after flicker cessation).

The effects of FL provocation on retinal vessel dilation are usually assessed using the parameters extracted from the RVA device software (the maximum and minimum dilation post flicker onset). Additionally baseline diameter fluctuation (peakto-trough amplitude prior to flicker onset), dilation amplitude (difference between maximum and minimum dilation), and time to reach maximum dilation can also be assessed and this set of five parameters is sometimes termed sequential diameter response analysis (SDRA) ${ }^{16}$; though note other forms of analysis have been attempted.9,17,18 The aim of this study was firstly to evaluate how FL duration affected the various SDRA parameters; for example, how is maximum dilation/ maximum constriction affected by flicker duration? The second goal was to evaluate individual flicker cycles and assess the variability in SDRA parameters across flicker cycles in protocols of different FL duration.

\section{Methods}

\section{Subjects}

Twelve healthy, young individuals (eight males; age range, 2645 years) without systemic or ocular abnormalities were included in this study. Exclusion criteria were age less than 18 years, connective tissue disease, cancer, stroke, diabetes mellitus (DM), hypertension (HT; ratio of systolic (SBP) and diastolic (DBP) blood pressure, SBP/DBP > 140/90 mm Hg), atrial fibrillation (AF), stroke, history of any ocular disease, history of neurologic diseases associated with loss of visual function, or any type of ocular surgery. Ethical approval was obtained from Aston University Ethics Committee. Written informed consent was received from all individuals taking part in the study. This study has been designed and conducted in accordance with the Declaration of Helsinki.

\section{Study Protocol}

A full history and examination took place to ensure that subjects were free from any disease as outlined in the exclusion criteria. All subjects were instructed to refrain from consuming caffeinated products, chocolate, drinking alcohol, and smoking on the study day. To the best of our knowledge none of the participants were taking any medication. All subjects underwent the test procedure as outlined below. Intraocular pressure was measured using noncontact tonometry (I-CARE; Mainline Instruments Ltd., Birmingham, UK). Systemic blood pressure was measured using a digital sphygmomanometer (Digital BP Monitor UA-767EX-C; PMS Instruments, Maidenhead, UK) following best practice procedures. ${ }^{19}$

Dynamic Retinal Vessel Assessment. After full pupil dilation was reached with $1 \%$ tropicamide (Chauvin Pharmaceuticals Ltd., Kingston-Upon-Thames, UK), vessel diameters of retinal arteries and veins were obtained using the Retinal Vessel Analyser (RVA; Imedos Systems, Jena, Germany). ${ }^{20}$

In brief, retinal vessel diameters in the patient's preferred eye were measured continuously at a sampling rate of $25 \mathrm{~Hz}$. Stimulation of retinal blood vessels occurred through optoelectronic interruption of the green fundus illumination used by the RVA resulting in a FL provocation of $12.5 \mathrm{~Hz}^{21}$ After BP stabilization and image focusing a vessel segment of the superior temporal retinal artery and vein was selected at a distance of 1.5 to 2 disc diameters (DD) away from the margins of the optic nerve head $(\mathrm{ONH})$. The baseline diameter of one retinal artery and one retinal vein was recorded for 50 seconds followed by three cycles of 5, 7, 10, or 20 second flicker provocation. Each flicker cycle was followed by 80 seconds recovery time. A minimum of 5 to 10 minutes rest between each duration was applied to ensure good fixation. See Figure 1 for details of all the FL durations tested. The order in which the FL durations were tested for each subject was chosen pseudorandomly by computer.

Sequential Diameter Response Analysis (SDRA). The relative diameter recordings (\% re baseline) were extracted 


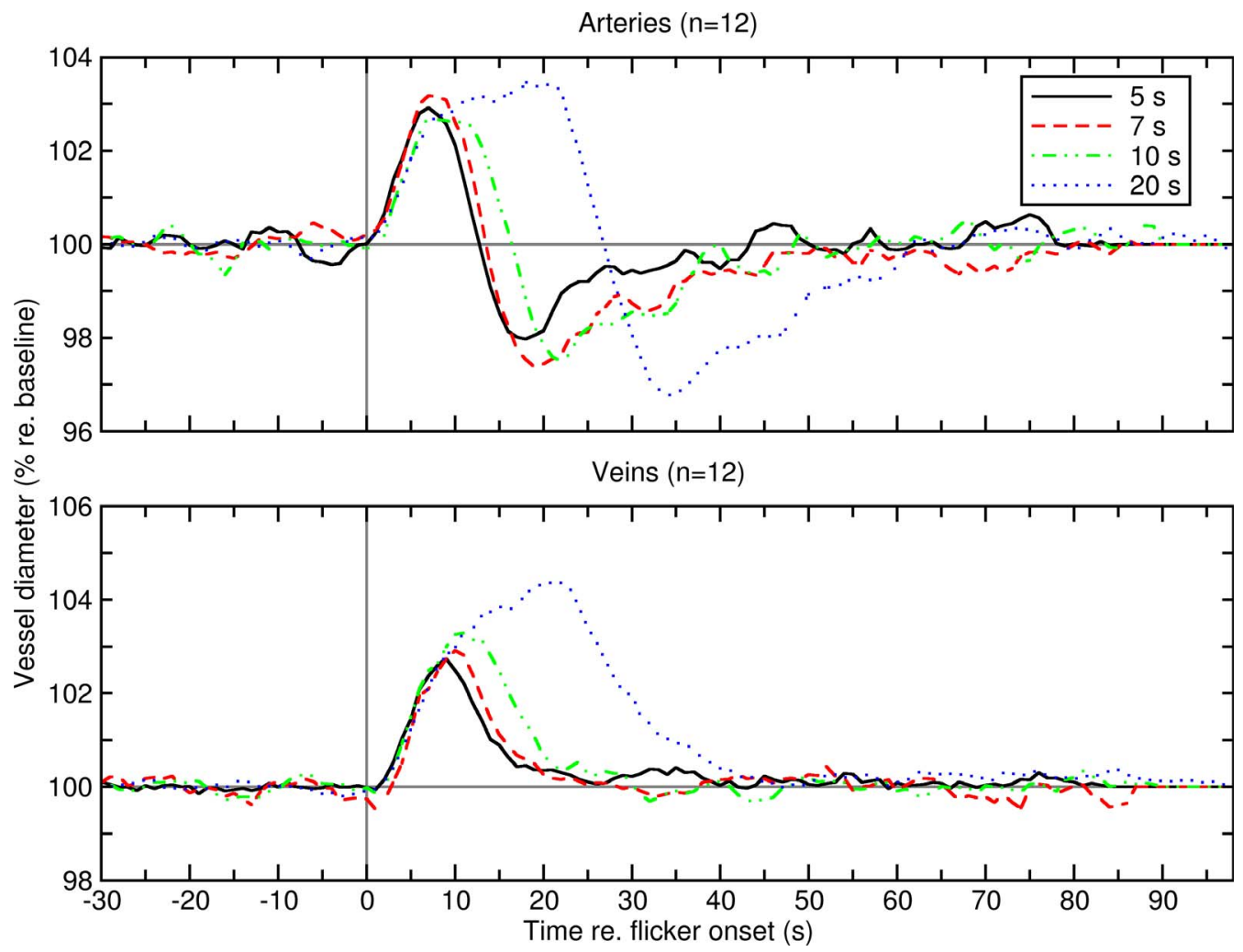

Figure 2. Each line represents the mean of 12 retinal vessel diameters of all 12 participants average flicker profiles for arteries (top panel) and veins (bottom panel) for each flicker duration $(5,7,10$, and 20 seconds). Vertical gray line denotes flicker onset; horizontal gray line denotes baseline diameter normalized to $100 \%$.

from the Imedos software. From these a number of standard measures were computed, known as SDRA, ${ }^{11,16}$ either for each individual flicker cycle or for the average of the three flicker cycles; baseline diameter fluctuation (BDF, \%), the difference between maximum and minimum diameter during 20 seconds prior to FL onset; maximum dilation (MD, \%), the maximum diameter attained between FL onset and 30 seconds after FL offset; maximum constriction (MC, \%), the minimum diameter attained from 5 seconds after FL onset to 30 seconds after FL offset; dilation amplitude (DA, \%), difference between MD and $\mathrm{MC}$; and reaction time (RT, seconds) or time to MD (seconds).

\section{Statistical Analysis}

For the statistical analysis, we used the software package IBM SPSS Statistics 20 (IBM Corp., Armonk, NY, USA). Two-way repeated-measures ANOVA was used to examine the differences between FL durations, individual flicker cycles and any interaction of the two. Additionally a series of repeatedmeasures 1-way ANOVAs were computed for parameters obtained from the flicker profiles averaged across all 3flicker-cycles (note that this is not the same as averaging the parameters from each flicker cycle individually). Where Mauchly's test of sphericity was significant Greenhouse-Geisser corrected degrees of freedom were used to compute $P$ values. All post hoc tests were performed according to Fisher's LSD at the $5 \%$ level. Effects sizes were reported in terms of $\eta^{2}$.

\section{Results}

All 12 participants were normotensive (mean $\mathrm{SBP}=110 \mathrm{~mm}$ $\mathrm{Hg}$, range, 87-122 $\mathrm{mm} \mathrm{Hg}$, mean $\mathrm{DBP}=68 \mathrm{~mm} \mathrm{Hg}$, range, 49$75 \mathrm{~mm} \mathrm{Hg}$ ) and exhibited normal IOP (IOP $=11.5 \mathrm{~mm} \mathrm{Hg}$, range, 8-14 $\mathrm{mm} \mathrm{Hg}$ ). All baseline diameter and dilatory parameters for retinal arterioles and veins can be found in Tables 1 and 2 , respectively. We confirmed that the vessel segment diameters were comparable at the start of each measurement (5-, 7-, 10-, and 20-second flicker duration) with a pair of 1-way repeated measures ANOVA; there was no significant difference between the absolute baseline diameters for either arteries $\left(F[3,33]=2.043, P=0.127, \eta^{2}=0.157\right)$ or veins $\left(F[3,33]=1.666, P=0.193, \eta^{2}=0.132\right)$. Baseline diameter fluctuation, a measure that can indicate if vessels have returned to resting state, was comparable across FL cycles for arteries $\left(F[3,33]=1.265 ; P=0.303, \eta^{2}=0.041\right)$ and veins $\left(F[3,33]=0.397 ; P=0.756, \eta^{2}=0.015\right)$ and across $\mathrm{FL}$ durations for arteries $\left(F[2,22]=0.070 ; P=0.933, \eta^{2}=0.001\right)$ and veins $\left(F[2,22]=1.654 ; P=0.214, \eta^{2}=0.022\right)$.

\section{Individual FL Cycles and FL Duration}

The mean vessel profiles for each duration are shown in Figure 2. For arteries (Fig. 2, upper panel) there is an initial rapid dilatory phase lasting 5 to 10 seconds before dilatory response plateaus (20 seconds, blue dotted curve). There is no systematic difference in peak diameter (MD) between the different FL durations. Upon FL offset the return to baseline appears to proceed at the same rate regardless of FL duration, though peak constriction (MC) appears to increase with FL duration.

For veins (Fig. 2, lower panel) there is an initial rapid dilatory phase lasting 5 to 10 seconds after which the dilatory response slows but still shows an increase (20 seconds, blue dotted curve). Peak diameter (MD) appears to increase with FL duration. Upon FL offset the return to baseline appears to proceed at the same rate regardless of FL duration. There is no 
TABLE 1. Arterial Parameters (Individual Flicker Cycles)

\begin{tabular}{|c|c|c|c|c|c|c|c|c|c|}
\hline \multirow[b]{2}{*}{ Parameter } & \multirow[b]{2}{*}{ Flicker Cycle } & \multicolumn{4}{|c|}{ Flicker Duration, s } & \multicolumn{4}{|c|}{ Statistics } \\
\hline & & $\begin{array}{c}5 \\
\text { Mean (SD) }\end{array}$ & $\begin{array}{c}7 \\
\text { Mean (SD) }\end{array}$ & $\begin{array}{c}10 \\
\text { Mean (SD) }\end{array}$ & $\begin{array}{c}20 \\
\text { Mean (SD) }\end{array}$ & & $\boldsymbol{F}$ & $P$ Values & $\eta^{2}$ \\
\hline Diameter, $\mu \mathrm{m}$ & & $112(19)$ & $113(18)$ & $114(19)$ & $112(19)$ & $F(3,33)$ & 2.043 & 0.127 & 0.157 \\
\hline $\mathrm{BDF}, \%$ & $\begin{array}{l}1 \\
2 \\
3\end{array}$ & $\begin{array}{l}4.6(2.3) \\
4.7(2.1) \\
4.4(1.8)\end{array}$ & $\begin{array}{l}4.1(1.9) \\
4.2(2.1) \\
4.9(3.1)\end{array}$ & $\begin{array}{l}4.1(1.9) \\
4.4(2.4) \\
3.8(1.5)\end{array}$ & $\begin{array}{l}4.1(1.7) \\
3.4(1.1) \\
3.9(1.5)\end{array}$ & $\begin{array}{l}F_{D}(3,33) \\
F_{C}(2,22) \\
F_{I}(6,66)\end{array}$ & $\begin{array}{l}1.265 \\
0.070 \\
0.946\end{array}$ & $\begin{array}{l}0.303 \\
0.933 \\
0.469\end{array}$ & $\begin{array}{l}0.041 \\
0.001 \\
0.038\end{array}$ \\
\hline $\mathrm{MD}, \%$ & $\begin{array}{l}1 \\
2 \\
3\end{array}$ & $\begin{array}{l}3.5(1.8) \\
3.8(2.0) \\
3.3(1.4)\end{array}$ & $\begin{array}{l}3.5(3.0) \\
3.5(1.6) \\
4.6(3.1)\end{array}$ & $\begin{array}{l}3.7(2.8) \\
3.5(1.7) \\
3.5(1.7)\end{array}$ & $\begin{array}{l}3.7(2.1) \\
4.2(1.6) \\
4.9(2.1)\end{array}$ & $\begin{array}{l}F_{D}(2.2,23.8) \\
F_{C}(2,22) \\
F_{I}(2.1,22.8)\end{array}$ & $\begin{array}{l}1.828 \\
0.668 \\
1.547\end{array}$ & $\begin{array}{l}0.180 \\
0.523 \\
0.234\end{array}$ & $\begin{array}{l}0.040 \\
0.016 \\
0.054\end{array}$ \\
\hline MC, $\%$ & $\begin{array}{l}1 \\
2 \\
3\end{array}$ & $\begin{array}{l}3.6(1.4) \\
2.8(1.2) \\
3.2(1.8)\end{array}$ & $\begin{array}{l}4.5(1.7) \\
3.0(1.5) \\
3.5(1.8)\end{array}$ & $\begin{array}{l}3.6(1.5) \\
3.2(1.7) \\
3.6(1.8)\end{array}$ & $\begin{array}{l}4.1(2.8) \\
3.9(2.3) \\
4.0(1.8)\end{array}$ & $\begin{array}{l}F_{D}(3,33) \\
F_{C}(2,22) \\
F_{I}(6,66)\end{array}$ & $\begin{array}{r}3.160 \\
20.458 \\
0.700\end{array}$ & $\begin{array}{r}\mathbf{0 . 0 3 7} \\
<\mathbf{0 . 0 0 1} \\
0.650\end{array}$ & $\begin{array}{l}\mathbf{0 . 1 0 5} \\
\mathbf{0 . 0 9 8} \\
0.023\end{array}$ \\
\hline DA, \% & $\begin{array}{l}1 \\
2 \\
3\end{array}$ & $\begin{array}{l}7.0(2.6) \\
6.6(2.6) \\
6.5(2.4)\end{array}$ & $\begin{array}{l}8.0(3.8) \\
6.5(2.7) \\
8.1(3.8)\end{array}$ & $\begin{array}{l}7.4(3.7) \\
6.7(2.6) \\
7.1(2.7)\end{array}$ & $\begin{array}{l}8.8(3.6) \\
8.1(2.7) \\
8.9(2.9)\end{array}$ & $\begin{array}{l}F_{D}(3,33) \\
F_{C}(2,22) \\
F_{I}(3.1,34.1)\end{array}$ & $\begin{array}{l}6.098 \\
1.728 \\
1.171\end{array}$ & $\begin{array}{l}\mathbf{0 . 0 0 2} \\
0.201 \\
0.302\end{array}$ & $\begin{array}{l}\mathbf{0 . 1 6 1} \\
0.034 \\
0.029\end{array}$ \\
\hline $\mathrm{RT}_{\mathrm{A}}, \mathrm{s}$ & $\begin{array}{l}1 \\
2 \\
3\end{array}$ & $\begin{array}{l}7.8(1.7) \\
7.4(2.3) \\
7.3(1.6)\end{array}$ & $\begin{array}{l}6.8(3.7) \\
8.1(1.7) \\
8.1(1.5)\end{array}$ & $\begin{array}{r}8.7(2.9) \\
11.3(4.9) \\
8.8(2.0)\end{array}$ & $\begin{array}{l}15.9(4.8) \\
15.9(4.1) \\
17.5(3.6)\end{array}$ & $\begin{array}{l}F_{D}(1.6,18.1) \\
F_{C}(1.2,13.2) \\
F_{I}(2.5,27.3)\end{array}$ & $\begin{array}{r}\mathbf{2 3 . 8 8 0} \\
0.313 \\
1.766\end{array}$ & $\begin{array}{r}<\mathbf{0 . 0 0 1} \\
0.626 \\
0.184\end{array}$ & $\begin{array}{l}\mathbf{0 . 3 6 2} \\
0.004 \\
0.046\end{array}$ \\
\hline
\end{tabular}

Values in parentheses after $F$ are the within- and between-subject degrees of freedom. Statistical values in bold are signficant at the 5\% level. mu, measurement units; $\mathrm{RT}_{\mathrm{A}}$, arterial reaction time (time to reach $\mathrm{MD}$ following flicker onset); $F_{D}$, between flicker-duration differences; $F_{c}$, between flicker-cycle differences; $F_{I}$, flicker duration and flicker cycle interaction. Statistical values in bold are signficant at the $5 \%$ level.

evidence of any constriction in venous diameters below baseline diameter.

In general, increasing the duration of the FL increased arterial MC, DA, and RT (Table 1; Fig. 3). The statistical significance of these effects was explored with a series of 2way (flicker duration $\times$ flicker cycle) repeated-measures ANOVA. There were significant main effects of flicker duration for arterial MC $\left(F_{D}[3,33]=3.160, P=0.037, \eta^{2}=0.105\right)$, arterial DA $\left(F_{D}[3,33]=6.098, P=0.002, \eta^{2}=0.161\right)$, and arterial RT $\left(F_{D}[1.6,18.1]=23.880, P<0.001, \eta^{2}=0.362\right)$ but there was no significant main effect of flicker duration for arterial $\mathrm{MD}\left(F_{D}[2.2,23.8]=1.828, P=0.180, \eta^{2}=0.040\right)$. There was a significant main effect of flicker cycle for arterial MC only $\left(F_{C}[2,22]=20.458, P<0.001, \eta^{2}=0.098\right)$. For arteries there were no significant interactions between flicker duration and flicker cycle for any of the measures tested.

To determine the source of these significant main effects a number of post hoc tests were performed. Pairwise comparisons indicated that the significant main effects of flicker duration on arterial MC, DA, and RT were driven by differences $(P<0.05)$ in the results between 20 seconds and two or more of the other durations. For arterial MC the significant effect of flicker cycle was driven by a significant difference between MC for the first cycle and the remaining cycles; arterial MC was significantly larger $(P<0.001)$ for the first cycle than either the second or the third.

Increasing the duration of the FL increased venous MD, DA, and RT (see Table 2; Fig. 4). The statistical significance of these effects was explored with a series of 2-way (flicker duration $\times$ flicker cycle) repeated measures ANOVA. There were significant main effects of flicker duration for venous $\mathrm{MD}\left(F_{D}[1.6\right.$, $\left.17.9]=10.159, P=0.002, \eta^{2}=0.249\right)$, venous DA $\left(F_{D}[3,33]=\right.$ 12.984, $\left.P<0.001, \eta^{2}=0.279\right)$, and venous $\mathrm{RT}\left(F_{D}[3,33]=\right.$ 86.549, $\left.P<0.001, \eta^{2}=0.547\right)$. For veins there were no significant main effects of flicker cycle or any significant interactions between flicker duration and flicker cycle for any of the measures tested.
Post hoc tests indicated that the main effects of flicker duration for venous MD and DA were driven by the results for both 10 and 20 seconds FL being significantly larger than those of 5 and 7 seconds; for both venous MD and DA there was no significant difference between 5 and 7 seconds $(P>0.05)$ or 10 and 20 seconds $(P>0.05)$. Venous MD and DA, however, at 10 or 20 seconds were significantly different from that at 5 or 7 seconds $(P>0.05)$. For venous RT only 5 and 7 seconds were not significantly different from each other $(P=0.771)$.

\section{Average of 3 Flicker Cycles and FL Duration}

For SDRA computed from the flicker-averaged readings there are statistically significant effects of flicker duration on arterial MC $\left(F[3,33]=4.880, P=0.006, \eta^{2}=0.307\right)$, arterial DA $(F[3$, $\left.33]=7.915, P<0.001, \eta^{2}=0.418\right)$, and arterial RT $(F[1.6$, $\left.17.1]=134.090, P<0.001, \eta^{2}=0.924\right)$. There was no statistically significant effect of duration for arterial MD $(F[3$, $33]=2.514, P=0.075, \eta^{2}=0.186$ ). There were statistically significant effects of flicker duration on venous MD ( $F[1.9$, $\left.20.5]=7.598, P=0.004, \eta^{2}=0.409\right)$, venous DA $(F[2.0,20.0]=$ 7.713, $\left.P=0.003, \eta^{2}=0.412\right)$, and venous RT $(F[2.0,21.5]=$ 48.445, $\left.P<0.001, \eta^{2}=0.815\right)$. There was no significant effect of duration on venous MC $(F[3,33]=0.434, P=0.730)$, see Table 3.

At durations of 5, 7, and 10 seconds arterial MD was, on average, $87 \%, 92 \%$, and $78 \%$, respectively, of that of MD at 20 seconds; the vast majority of arterial dilatory capacity is reached within 5 to 10 seconds. The dip in percentage at 10 seconds relative to 5 and 7 seconds is likely due to variability in the data rather than a real effect because the dip corresponds to less than $1 \%$ of arterial diameter and the $\mathrm{SD}$ of $\mathrm{MD}$ is approximately $2 \%$.

\section{Discussion}

In this work, we systematically investigated the effects of flicker duration on arterial and venous diameter response. The 

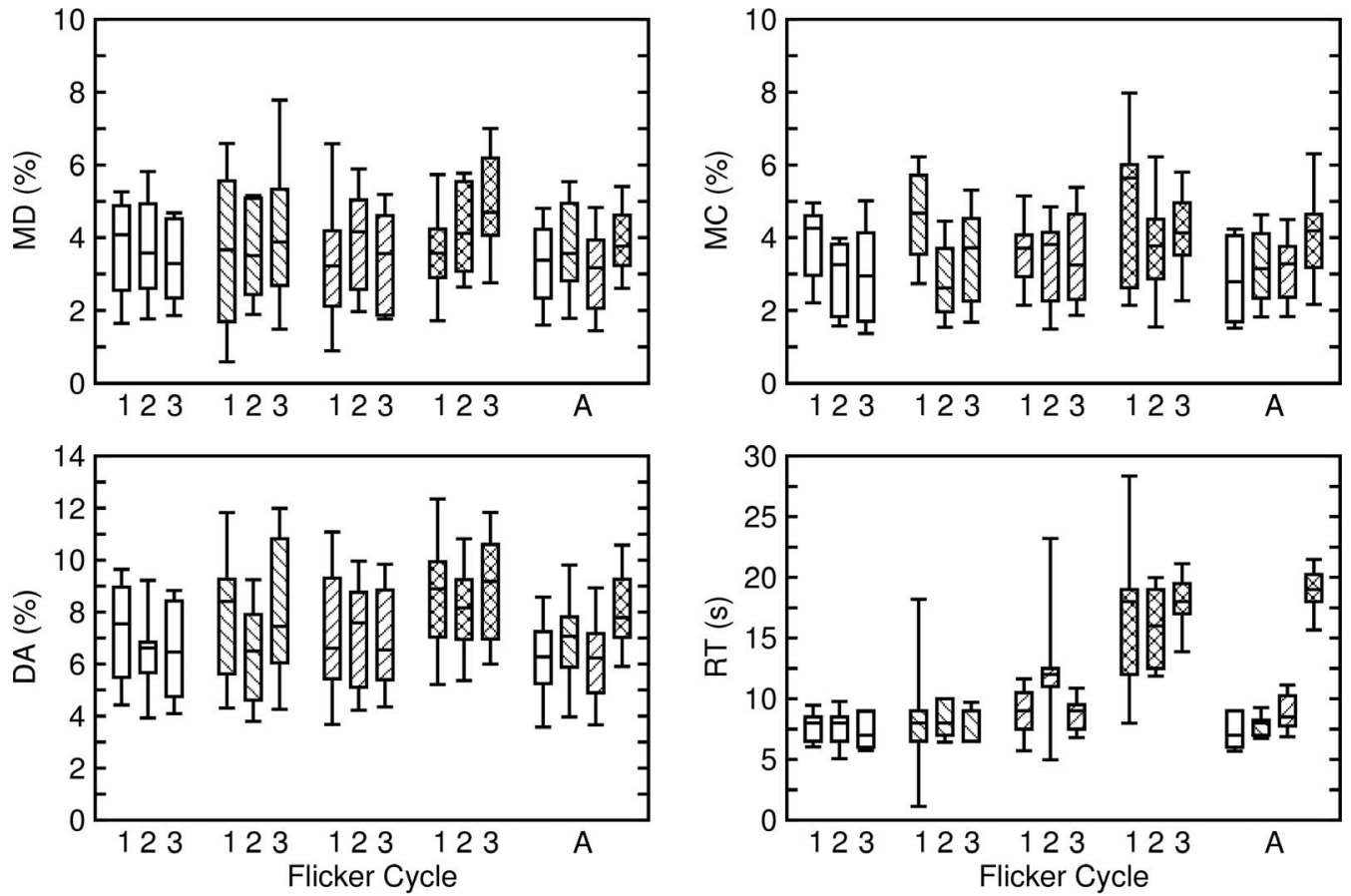

Flicker duration:

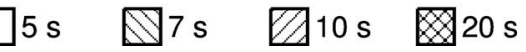

Figure 3. Sequential diameter response analysis for Arteries with (1) MD, (2) MC, (3) DA, and (4) RT for each individual flicker cycle (1, 2, 3) and from the average flicker profile (A) for flicker durations of 5 (white bars), 7 (left-oblique), 10 (right-oblique), and 20 seconds (cross hatched). The borizontal line, box, and whiskers represent, respectively, the median, interquartile range, and mean \pm 1 SD. For RT, one subject at flicker cycle 1 for 7 seconds ( $\mathrm{RT}=35$ seconds) and 20 seconds ( $\mathrm{RT}=47$ seconds) and a different subject for flicker cycle 2 at 10 seconds (RT $=39$ seconds) cause the large error bars. In each case these are a result of no or relatively little dilation during the flicker phase and baseline diameter fluctuation exceeding any residual dilation once flicker has ceased.

results confirm previous observations in humans ${ }^{15}$ and animals $^{22}$ that most of the arterial dilation occurs within the first 10 seconds of stimulation. Additionally no significant increase in maximum dilation was found in arteries between flicker durations of 5, 7, 10, and 20 seconds. A significant increase in both dilation amplitude and maximum constriction was found for arteries. The maximum dilation and dilation amplitude for veins were found to significantly increase with

TABle 2. Venous Parameters (Individual Flicker Cycles)

\begin{tabular}{|c|c|c|c|c|c|c|c|}
\hline \multicolumn{4}{|c|}{ Flicker Duration, s } & \multicolumn{4}{|c|}{ Statistics } \\
\hline 5 & 7 & 10 & 20 & & & & \\
\hline Mean (SD) & Mean (SD) & Mean (SD) & Mean (SD) & & $\boldsymbol{F}$ & $P$ Values & $\eta^{2}$ \\
\hline $141(15)$ & $142(15)$ & $143(16)$ & $142(17)$ & $F(3,33)$ & 1.666 & 0.193 & 0.132 \\
\hline $2.3(0.9)$ & $2.7(1.3)$ & $2.0(0.9)$ & $2.4(1.1)$ & $F_{D}(3,33)$ & 0.397 & 0.756 & 0.015 \\
\hline $2.5(0.9)$ & $2.1(0.9)$ & $2.6(1.7)$ & $2.6(1.3)$ & $F_{C}(2,22)$ & 1.654 & 0.214 & 0.022 \\
\hline $2.5(0.9)$ & $3.4(2.8)$ & $2.6(0.8)$ & $2.5(0.9)$ & $F_{I}(2.5,27.1)$ & 1.691 & 0.199 & 0.054 \\
\hline $2.7(1.6)$ & $3.3(2.4)$ & $3.9(2.6)$ & $4.7(2.1)$ & $F_{D}(1.6,17.9)$ & 10.159 & 0.002 & 0.249 \\
\hline $3.4(2.1)$ & $3.7(2.2$ & $4.1(2.6)$ & $5.6(2.5)$ & $F_{C}(2,22)$ & 1.874 & 0.177 & 0.024 \\
\hline $3.2(2.2)$ & $3.4(1.7)$ & $4.2(2.6)$ & $5.4(2.5)$ & $F_{I}(6,66)$ & 0.234 & 0.964 & 0.007 \\
\hline $0.5(0.7)$ & $1.0(0.8)$ & $1.1(1.2)$ & $1.1(1.2)$ & $F_{D}(3,33)$ & 0.849 & 0.477 & 0.017 \\
\hline $0.6(0.5)$ & $0.5(0.8)$ & $0.5(1.0)$ & $1.1(1.2)$ & $F_{C}(2,22)$ & 1.609 & 0.223 & 0.029 \\
\hline $0.8(0.8)$ & $0.8(0.7)$ & $1.2(1.0)$ & $1.1(1.2)$ & $F_{I}(3.8,41.7)$ & 0.499 & 0.727 & 0.023 \\
\hline $3.2(1.7)$ & $4.3(2.2)$ & $5.0(3.2)$ & $5.8(2.5)$ & $F_{D}(3,33)$ & 12.984 & $<0.001$ & 0.279 \\
\hline $4.1(2.1)$ & 4.3 (1.9) & $4.6(2.5)$ & $6.7(3.0)$ & $F_{C}(2,22)$ & 0.949 & 0.402 & 0.014 \\
\hline $4.1(1.9)$ & $4.2(1.5)$ & $5.4(2.7)$ & $6.5(2.7)$ & $F_{I}(6,66)$ & 0.699 & 0.651 & 0.019 \\
\hline $10.3(4.6)$ & $9.5(2.0)$ & $11.1(5.1)$ & $20.7(2.1)$ & $F_{D}(3,33)$ & 86.549 & $<0.001$ & 0.547 \\
\hline $9.7(3.6)$ & 9.0 (1.9) & $12.0(3.5)$ & $20.6(4.9)$ & $F_{C}(2,22)$ & 1.053 & 0.366 & 0.008 \\
\hline $9.1(1.9)$ & $9.8(3.6)$ & $12.2(5.1)$ & $19.1(3.9)$ & $F_{I}(2.2,23.9)$ & 0.768 & 0.485 & 0.019 \\
\hline
\end{tabular}

Values in parentheses after $F$ are the within- and between-subject degrees of freedom. Statistical values in bold are signficant at the 5\% level. 

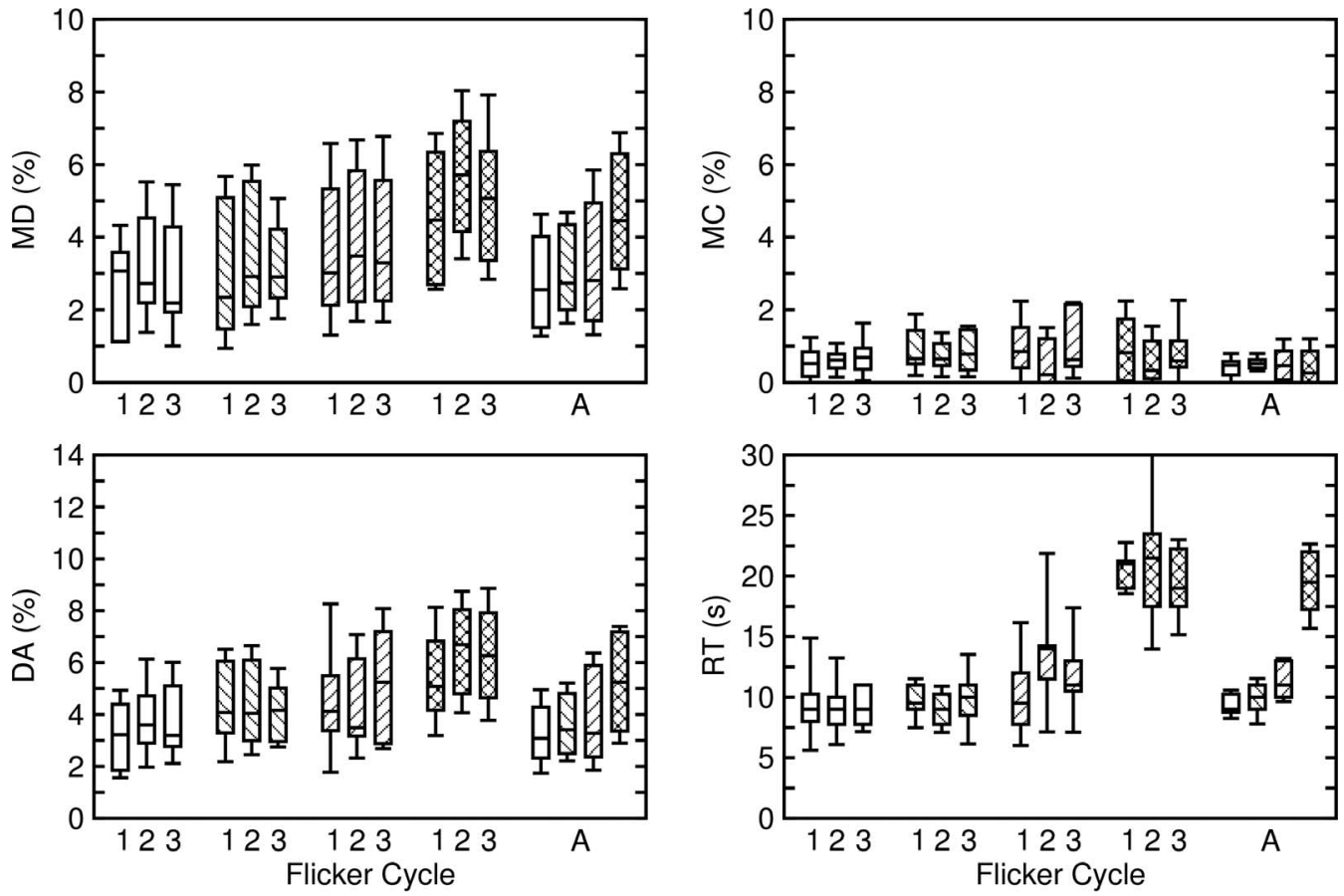

Flicker duration:

$\square 5 \mathrm{~s}$

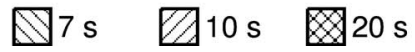

Figure 4. Sequential diameter response analysis for veins with (1) MD, (2) MC, (3) DA, and (4) RT for each individual flicker cycle (1, 2, 3) and from the average flicker profile (A) for flicker durations of 5 seconds (white bars), 7 seconds (left-oblique), 10 seconds (right-oblique), and 20 seconds (cross batched). The borizontal line, box, and whiskers represent, respectively, the median, interquartile range, and mean \pm 1 SD.

increasing flicker duration. Although there has been prior research aimed at determining the best FL frequency, ${ }^{13}$ effects of illumination, ${ }^{15}$ and some comparison between two flicker durations ${ }^{15}$ there has, as far as we know, been no systematic effort made to examine the effects of shorter FL durations and hence the time-course of retinal vessel dilation and constriction due to FL provocation as a function of FL duration.

Although we found no significant effect of FL duration on arterial MD in our sample and the effect size was small $\left(\eta^{2}=\right.$ 0.04 ) it is likely that in a much larger sample a significant effect would have been found since for the flicker profile averaged over three cycles the effect size was larger $\left(\eta^{2}=0.186\right)$. This, however, does not detract from the main finding that the majority of arterial dilation occurs by 10 seconds, which would likely be confirmed by studies on larger samples given that there appears to be no increase in variance in the SDRA parameters with decreasing flicker duration. One participant of extremely slight build had low blood pressure (SBP/DBP $=$ $87 / 49 \mathrm{~mm} \mathrm{Hg}$ ). As the effects of low blood pressure alone on retinal vessel reactivity are unknown we reran the statistical analysis without this participant's data and there was no change in the statistical significance of any of the effects.

That the vast majority of arterial dilation occurs within 5 to 10 seconds of FL onset in healthy vessels has many important

TABle 3. Arterial and Venous Parameters (Averaged Across Flicker Cycles)

\begin{tabular}{|c|c|c|c|c|c|c|c|c|}
\hline \multirow[b]{3}{*}{ Parameter } & \multicolumn{4}{|c|}{ Flicker Duration, s } & \multicolumn{4}{|c|}{ Statistics } \\
\hline & 5 & 7 & 10 & 20 & & & & \\
\hline & Mean (SD) & Mean (SD) & Mean (SD) & Mean (SD) & & $\boldsymbol{F}$ & $P$ Values & $\eta_{p}^{2}$ \\
\hline \multicolumn{9}{|l|}{ Arteries } \\
\hline BDF, \% & $2.4(1.2)$ & $2.7(1.4)$ & $2.4(1.1)$ & $2.3(0.8)$ & $F(3,33)$ & 0.446 & 0.722 & 0.039 \\
\hline $\mathrm{MD}, \%$ & $3.2(1.6)$ & 3.7 (1.9) & $3.1(1.7)$ & $4.0(1.4)$ & $F(3,33)$ & 2.514 & 0.075 & 0.186 \\
\hline MC, \% & $2.9(1.4)$ & $3.2(1.4)$ & $3.2(1.3)$ & $4.2(2.1)$ & $F(3,33)$ & 4.880 & 0.006 & 0.307 \\
\hline DA, \% & $6.1(2.5)$ & $6.9(2.9)$ & $6.3(2.6)$ & $8.2(2.3)$ & $F(3,33)$ & 7.915 & $<0.001$ & 0.418 \\
\hline $\mathrm{RT}, \mathrm{s}$ & $7.4(1.7)$ & $8.0(1.3)$ & $9.0(2.1)$ & $18.6(2.9)$ & $F(1.6,17.1)$ & 134.090 & $<0.001$ & 0.924 \\
\hline \multicolumn{9}{|l|}{ Veins } \\
\hline BDF, \% & $1.5(0.6)$ & $1.6(0.9)$ & $1.4(0.5)$ & $1.6(0.7)$ & $F(3,33)$ & 0.179 & 0.910 & 0.016 \\
\hline $\mathrm{MD}, \%$ & $3.0(1.7)$ & $3.2(1.5)$ & $3.6(2.3)$ & $4.7(2.1)$ & $F(1.9,20.5)$ & 7.598 & 0.004 & 0.409 \\
\hline MC, \% & $0.4(0.4)$ & $0.6(0.2)$ & $0.5(0.3)$ & $0.4(0.8)$ & $F(3,33)$ & 0.434 & 0.730 & 0.038 \\
\hline DA, \% & $3.3(1.6)$ & $3.7(1.5)$ & $4.1(2.3)$ & $5.1(2.2)$ & $F(2.0,22.0)$ & 7.713 & 0.003 & 0.412 \\
\hline RT, s & $9.4(1.2)$ & 9.7 (1.9) & $11.4(1.8)$ & $19.2(3.5)$ & $F(2.0,21.5)$ & 48.445 & $<0.001$ & 0.815 \\
\hline
\end{tabular}

Values in parentheses after $F$ are the within- and between-subject degrees of freedom. Statistical values in bold are signficant at the 5\% level. 
consequences for clinical work. Previous work that has attempted to stratify vascular diseases based on retinal vessel reactivity has had limited clinical use $^{11}$ because although reduced retinal vessel reactivity has been found in, for example, glaucoma ${ }^{8-10}$ or coronary artery disease ${ }^{7}$ there is often substantial overlap in SDRA parameters between healthy and clinical populations and stratification is only possible at a (large) group level. This is likely because 20 seconds of provocation begins to saturate the arterial response in both healthy and clinical groups reducing parametric differences between the vessel reactions. The parametric measurements currently available are also extremely limited and alternatives to SDRA, such as area-under-curve analysis ${ }^{9}$ and spectral analysis $^{17,18}$ are much less established and seem to show similar degrees of overlap between clinical groups. A comprehensive functional or descriptive model of the entire vessel reaction process to FL provocation is lacking but would likely provide better means of assessing the reaction profile in different clinical groups. Even if such a model is developed measuring at two flicker durations (e.g., 7 and 20 seconds) with three cycles each may be a better approach than measuring a single duration that begins to saturate the arterial reaction. However, some patients can struggle to maintain fixation during 20 seconds of FL provocation so measurements taken at a shorter duration only may provide better quality data; though the advantages of taking measurements at a single shorter FL duration must be balanced against the loss of information that might otherwise be gleaned from longer FL durations. The results presented here show that it is possible to obtain reliable reactions to short duration FL provocation in healthy subjects (note there is no suggestion of increased variance in SDRA measures as a function of decreasing flicker duration, see Tables 1 and 2), but whether the same is true for patients is an open question.

Our results show for the first time that although there are differences in some SDRA parameters for veins and arteries as FL duration increases this is not universal and, in particular, the vast majority of the retinal arterial dilatory capacity appears to be reached in 5 to 10 seconds. Furthermore, with the exception of RT we find no significant differences between flicker durations of 10 and 20 seconds. While the data for the four flicker durations provides sufficient evidence that arterial vessel dilation to FL stimulation has begun to saturate within approximately 10 seconds, venous dilation increases more strongly with increasing FL duration. In order to gain further insight into the dilatory response we ran a single cycle of 50 seconds FL duration on a subset of seven individuals who volunteered to return. This experiment (see Supplementary Material) revealed that in our subgroup arterial saturation has occurred well within 50 seconds FL duration since arterial MD (5.2\%) occurred well before the end of flicker offset ( $\mathrm{RT}=33.1$ seconds). Venous RT was, however, 42.1 seconds, indicating that veins continued to dilate even after arterial dilation had saturated. The difference between the arterial and venous RT could be due to the differences in anatomical structure between the two vessel types. Arterioles have thicker more muscular and contractile walls compared with those of venules, therefore they are actively involved in regulating vascular tone and blood flow. Venules lack this contractility and have a more passive role in the vascular system that is characterized by the delay in dilatory response in comparison with arteries (i.e., pulse delay ${ }^{23}$ and longer reaction times). ${ }^{6}$ During prolonged FL stimulation arterial dilation saturates but metabolic demand is sustained and blood flow increases. If this increase in blood flow cannot be achieved by arterial vessel dilation (blood flow is dependent on lumen size and blood velocity) then blood velocity in the arteries must increase (e.g., work by Michelson and colleagues ${ }^{24}$ reveals increased blood velocity in arteries during FL provocation). There is indirect evidence for this hypothesis from our results at FL durations of 50 seconds where venous RT (42.1 seconds) is much greater than arterial RT (33.1 seconds) indicating that the venous system is still draining more blood despite the arterial diameter saturation.

In conclusion, MD of retinal arteries for shorter FL durations ( $\leq 10$ seconds) is approximately $80 \%$ of MD for 20 seconds FL duration and in our sample there was no significant effect of FL duration. The results from a subset of seven subjects at 50 seconds FL duration indicate that arterial diameter saturates at approximately 30 seconds ( $\mathrm{RT}=33.1$ seconds). We did, however, find a robust significant effect of FL duration on arterial MC. In contrast with arteries we found a significant effect of FL duration on venous MD. Between-flicker-cycle differences in arterial MC underline the importance of analysing individual flicker cycles rather than their average in case they are not comparable.

\section{Acknowledgments}

Portions of this work have been presented at the annual meeting of the Association for Research in Vision and Ophthalmology, Fort Lauderdale, Florida, United States May 6-9, 2012 and EVER, Nice, France, October 10-14, 2012.

Disclosure: R. Heitmar, None; R.J. Summers, None

\section{References}

1. Garhöfer G, Zawinka C, Resch H, et al. Reduced response of retinal vessel diameters to flicker stimulation in patients with diabetes. Br J Ophthalmol. 2004;88:887-891.

2. Mandecka A, Dawczynski J, Blum M, et al. Influence of flickering light on the retinal vessels in diabetic patients. Diabetes Care. 2007;30:3048-3052.

3. Mandecka A, Dawczynski J, Vilser W, et al. Abnormal retinal autoregulation is detected by provoked stimulation with flicker light in well-controlled patients with type 1 diabetes without retinopathy. Diabetes Res Clin Pract. 2009;86:51-55.

4. Lim LS, Ling LH, Ong PG, et al. Dynamic responses in retinal vessel caliber with flicker light stimulation in eyes with diabetic retinopathy. Invest Ophthalmol Vis Sci. 2014;55: 5207-5213.

5. Lasta M, Pemp B, Schmidl D, et al. Neurovascular dysfunction precedes neural dysfunction in the retina of patients with type 1 diabetes. Invest Ophthalmol Vis Sci. 2013;54:842-847.

6. Heitmar R, Cubbidge RP, Lip GY, Gherghel D, Blann AD. Altered blood vessel responses in the eye and finger in coronary artery disease. Invest Ophthalmol Vis Sci. 2011;52: 6199-6205.

7. Al-Fiadh AH, Wong TY, Kawasaki R, et al. Usefulness of retinal microvascular endothelial dysfunction as a predictor of coronary artery disease. Am J Cardiol. 2015;115:609-613.

8. Garhöfer G, Zawinka C, Resch H, Huemer KH, Schmetterer L, Dorner GT. Response of retinal vessel diameters to flicker stimulation in patients with early open angle glaucoma. $J$ Glaucoma. 2004;13:340-344.

9. Gugleta K, Kochkorov A, Waldmann N, et al. Dynamics of retinal vessel response to flicker light in glaucoma patients and ocular hypertensives. Graefes Arch Clin Exp Ophthalmol. 2012;250:589-594.

10. Gugleta K, Türksever C, Polunina A, Orgül S. Effect of ageing on the retinal vascular responsiveness to flicker light in glaucoma patients and in ocular hypertension. Br J Ophthalmol. 2013;97:848-851. 
11. Heitmar R, Summers RJ. Assessing vascular function using dynamic retinal diameter measurements: a new insight on the endothelium. Thromb Haemost. 2012;107:1019-1026.

12. Garhofer G, Bek T, Boehm AG, et al. Use of the retinal vessel analyzer in ocular blood flow research. Acta Opbthalmol. 2010;88:717-722.

13. Polak K, Schmetterer L, Riva CE. Influence of flicker frequency on flicker-induced changes of retinal vessel diameter. Invest Opbthalmol Vis Sci. 2002;43:2721-2726.

14. Jeppesen P, Gregersen PA, Bek T. The age-dependent decrease in the myogenic response of retinal arterioles as studied with the Retinal Vessel Analyzer. Graefes Arch Clin Exp Ophthalmol. 2004;242:914-919.

15. Kotliar KE, Vilser W, Nagel E, Lanzl IM. Retinal vessel reaction in response to chromatic flickering light. Graefes Arch Clin Exp Opbthalmol. 2004;242:377-392.

16. Heitmar R, Blann AD, Cubbidge RP, Lip GY, Gherghel D. Continuous retinal vessel diameter measurements: the future in retinal vessel assessment? Invest Opbthalmol Vis Sci. 2010; 51:5833-5839.

17. Kotliar KE, Lanzl IM, Schmidt-Trucksäss A, et al. Dynamic retinal vessel response to flicker in obesity: a methodological approach. Microvasc Res. 2011;81:123-128.
18. Bek T, Jeppesen P, Kanters JK. Spontaneous high frequency diameter oscillations of larger retinal arterioles are reduced in type 2 diabetes mellitus. Invest Ophthalmol Vis Sci. 2013;54: 636-640.

19. Williams B, Poulter NR, Brown MJ, et al; for the British Hypertension Society. Guidelines for management of hypertension: report of the fourth working party of the British Hypertension Society, 2004-BHS IV. J Hum Hypertens. 2004; 18:139-185.

20. Vilser W, Nagel E, Lanzl I. Retinal Vessel Analysis - new possibilities. Biomed Tech (Berl). 2002;47(suppl 1 pt 2):682685.

21. Nagel E, Vilser W, Fink A, Riemer T. Variance of retinal vessel diameter response to flicker light. A methodical clinical study. Ophthalmologe. 2006;103:114-119.

22. Riva CE, Logean E, Falsini B. Visually evoked hemodynamical response and assessment of neurovascular coupling in the optic nerve and retina. Progr Retin Eye Res. 2005;24:183-215.

23. Michelson G, Harazny J. Relationship between ocular pulse pressures and retinal vessel velocities. Ophthalmology. 1997; 104:664-671.

24. Michelson G, Patzelt A, Harazny J. Flickering light increases retinal blood flow. Retina. 2002;22:336-343. 